\title{
Mechanical restriction versus human overreaction triggering congested traffic states
}

\author{
Hyun Keun Lee, ${ }^{1}$ Robert Barlovic, ${ }^{2}$ Michael Schreckenberg, ${ }^{2}$ and Doochul Kim ${ }^{1}$ \\ ${ }^{1}$ School of Physics, Seoul National University, Seoul 151-747, Korea and \\ ${ }^{2}$ Theoretische Physik Fakultät 4, Universität Duisburg-Essen, D-47048 Duisburg, Germany
}

(Dated: November 10, 2018)

\begin{abstract}
A new cellular automaton (CA) traffic model is presented. The focus is on mechanical restrictions of vehicles realized by limited acceleration and deceleration capabilities. These features are incorporated into the model in order to construct the condition of collision-free movement. The strict collision-free criterion imposed by the mechanical restrictions is softened in certain traffic situations, reflecting human overreaction. It is shown that the present model reliably reproduces most empirical findings including synchronized flow, the so-called pinch effect, and the time-headway distribution of free flow. The findings suggest that many free flow phenomena can be attributed to the platoon formation of vehicles (platoon effect).

PACS numbers: 89.40.-a, 45.70.Vn, 05.45.-a, 05.20.Dd
\end{abstract}

Traffic flow phenomena have been analyzed and modelled from the viewpoint of statistical physics since the early 1990s. The main issues are the characterization of traffic phases and transitions among them as well as the development of appropriate traffic models. The investigation of empirical data has lead to the identification of three traffic phases (free, synchronized, jammed) 1, 2, 3, 4, 5, 6, 7]. Furthermore, time-headway distributions extracted from single vehicle data have been reported [8], showing that even free flow is not as trivial as previously believed. In order to reproduce the empirical findings, many traffic models have been proposed both from the microscopic 9, 10, 11] and the macroscopic 12] viewpoints. Also, there are efforts to establish a link between them 13, 14, 15, 16] in order to probe any universal features. These achievements have deeply influenced the understanding of traffic flow far from equilibrium with interacting particles showing collective behaviors.

A primary objective of traffic models is to explain the synchronized flow phase that is characterized by a considerably high flux without any clear density-flux relation. Unfortunately, most models produce merely the free flow and jammed traffic. The fluctuating profiles, appearing during the transient process heading towards the phase separation of the two phases above, are regarded as the synchronized flow in those models. However, such a behavior is not compatible with the stability of the synchronized flow found in empirical data. The approach of Knospe et al. 17] produces a different type of solution for the synchronized flow, which is not a part of any transient process. However, the single vehicle data show unrealistic velocity fluctuations in this solution. Recently, Kerner et al. have claimed to be able to reproduce the synchronized flow 18]. However, in their model, a phenomenological requirement for synchronized flow is directly implemented, namely the driver's attempt to adopt the velocity of the vehicle in front within the so-called synchronization distance. Another shortcoming of existing traffic models is that even the free flow is not reproduced so successfully. In [8], it is reported that a small time-headway below $1 \mathrm{sec}$ is frequently observed especially in the free flow. Simple tuning of the spatiotemporal scale to achieve such a small time-headway results in an extraordinarily high flux. A method to bypass this problem is to utilize the gap between the next two vehicles in front [17], leading to a time-headway definitely below $1 \mathrm{sec}$. However, the coupling of two vehicles is inevitable in this approach, which is not observed empirically [19]. In this Letter, a new Cellular Automaton (CA) traffic model is presented, which overcomes the problems stated above. The mechanical restriction is introduced in the form of limited acceleration and deceleration capabilities. Also, human behavior is modelled as the driver's excessive response to the local traffic conditions. The former is a natural consequence of given physical limitations while the latter is incorporated into the model to reflect human overreaction. In the following, all variables are assumed to be integer numbers unless stated otherwise.

First, we introduce the limited capability of acceleration $(a)$ and deceleration $(D)$. For simplicity, these are both assumed to be constant. The idea of limited acceleration is implemented in most existing CA traffic models. However, the deceleration limitation has not been enforced strictly or systematically. It is important to note that the bounded braking capability changes the collision-free mechanism entirely. Most CA traffic models impose a collision-free condition explicitly by assigning arbitrary deceleration values required to prevent collision. However, it is rather natural to view the collisionfree flow as a consequence of moderate driving instead of infinite braking capabilities. For such a physically realizable flow, we first design a heuristic collision-free driving dynamics strictly observing the limited deceleration. The starting point of the new $\mathrm{CA}$ model is an inequality which guarantees safe driving. Here a vehicle prepares for the worst case, namely that the leading vehicle may brake suddenly at any time $t$. Since the follower's reaction is 
delayed due to the response time which is assumed to be the unit time of the model, whether secure driving is possible or not is determined at time $t+1$ when the reaction begins. The velocity which allows safe movement, represented by $c_{n}^{t+1}$ below, should satisfy

$x_{n}^{t}+\Delta+\sum_{i=0}^{\tau_{\mathrm{f}}\left(c_{n}^{t+1}\right)}\left(c_{n}^{t+1}-D i\right) \leq x_{n+1}^{t}+\sum_{i=1}^{\tau_{1}\left(v_{n+1}^{t}\right)}\left(v_{n+1}^{t}-D i\right)$,

where $x_{n}^{t}\left(v_{n}^{t}\right)$ is the location (velocity) of the $n$-th vehicle at time $t$ and the increased index represents the vehicle in front. $\Delta$ is the minimal coordinate difference required by the follower to guarantee its safety, and thus assumed to be at least the length of a vehicle $L$. Each summation accounts for successive decelerations during time steps $i=0,1, . ., \tau_{\mathrm{f}}\left(i=1, . ., \tau_{\mathrm{l}}\right)$ with maximum braking capability $D$, where $\tau_{\mathrm{f}}\left(\tau_{\mathrm{l}}\right)$ for the follower (leader) will be specified below. The zero-based summation index stands for the response time of the follower. For $\tau_{\mathrm{f}, 1}(v)=v / D$ and $\Delta=L$, Eq. (10) suggests such $c_{n}^{t+1}$ that can guarantee a complete stop showing bumper-tobumper configuration. We call this dynamics the strict collision-free dynamics (or criterion) in this work. Later on, the expressions for $\tau_{\mathrm{f}, 1}$ and $\Delta$ will be modified to take into account the human overreaction. Note that for a given $n$, the safe velocity $c_{n}^{t+1}$ is not unique but just has an upper bound. In the following, the largest $c_{n}^{t+1}$ satisfying Eq. (1), denoted by $\tilde{c}_{n}^{t+1}$, is used to reflect the desire of drivers to move as fast as possible.

Next, an element of human behavior is introduced which is actually responsive to the local traffic situation. Generally, it is accepted that a driver's maneuvers are not precisely predictable by a simple rule. The usual solution for this problem in simulation models is to introduce fluctuations which cover the human factor in a stochastic way. However, a different strategy is adopted here. It is supposed that the driver's behavior may be biased depending on the local traffic situation. To implement this simply, a 2-state variable is introduced:

$$
\gamma_{n}^{t}= \begin{cases}0 & \text { for } v_{n}^{t} \leq v_{n+1}^{t} \leq v_{n+2}^{t} \text { or } v_{n+2}^{t} \geq v_{\text {fast }}, \\ 1 & \text { otherwise }\end{cases}
$$

with a constant $v_{\text {fast }}$ slightly below $v_{\max }$. The state $\gamma_{n}^{t}=$ 0 corresponds to a situation where the driver judges that the local situation is optimistic since the cars in front are speeding away. It is assumed that the driver will move faster in order to catch the car ahead, even faster than the allowed velocity under the strict collision-free dynamics. This state is denoted as the optimistic state. Otherwise, for $\gamma_{n}^{t}=1$, the driver is in the defensive state, where the vehicle slows down below the velocity due to the strict collision-free criterion. This distinction in respect to the local traffic situation is termed human overreaction here. It is realized in the model by manipulating $\tau_{\mathrm{f}, \mathrm{l}}(v)$ and $\Delta$ in (1) as follows:

$\Delta=L+\gamma_{n}^{t} \max \left\{0, \min \left\{g_{\text {add }}, v_{n}^{t}-g_{\text {add }}\right\}\right\}$, $\tau_{\mathrm{f}}(v)=\gamma_{n}^{t} v / D+\left(1-\gamma_{n}^{t}\right) \max \left\{0, \min \left\{v / D, t_{\text {safe }}\right\}-1\right\}$, $\tau_{1}(v)=\gamma_{n}^{t} v / D+\left(1-\gamma_{n}^{t}\right) \min \left\{v / D, t_{\mathrm{safe}}\right\}$.

Herein $g_{\text {add }}$ is introduced for an additional security gap in the defensive state $\left(\gamma_{n}^{t}=1\right)$ and $t_{\text {safe }}$ is a maximal time step during which the follower observes his/her own safety in the optimistic state. The additional -1 for $\tau_{\mathrm{f}}(v)$ compensates for the surplus time step due to the follower's response time only when $\gamma_{n}^{t}=0$, and thus the role of $t_{\text {safe }}$ is properly implemented. For $\gamma_{n}^{t}=0$, the $\tau_{\mathrm{f}, 1}$ can be smaller than those $(v / D)$ necessary for complete stops while $\Delta$ returns to $L$. Consequently, lower safety is required compared to that needed by strict collision-free dynamics, and thus a faster $\tilde{c}_{n}^{t+1}$ can be chosen. On the other hand, for $\gamma_{n}^{t}=1, \Delta$ can be larger than $L$ while $\tau_{\mathrm{f}, 1}(v)$ return to $v / D$, which implies over safety. In this way, a lower $\tilde{c}_{n}^{t+1}$ can be assigned.

The update rules of the model can be written in the following form:

$$
\begin{aligned}
& 1 p=\max \left\{p_{\mathrm{d}}, p_{0}-v_{n}^{t}\left(p_{0}-p_{\mathrm{d}}\right) / v_{\text {slow }}\right\} \\
& \left.2 \tilde{c}_{n}^{t+1}=\max \left\{c_{n}^{t+1} \mid c_{n}^{t+1} \text { satisfies Eqs. (10] }\right\}\right\} \\
& 3 \tilde{v}_{n}^{t+1}=\min \left\{v_{\max }, v_{n}^{t}+a, \max \left\{0, v_{n}^{t}-D, \tilde{c}_{n}^{t+1}\right\}\right\} \\
& 4 v_{n}^{t+1}=\max \left\{0, v_{n}^{t}-D, \tilde{v}_{n}^{t+1}-\eta\right\} \\
& \quad \text { where } \eta=1 \text { if } \operatorname{rand}()<p, \text { or } 0 \text { otherwise } \\
& 5 x_{n}^{t+1}=x_{n}^{t}+v_{n}^{t+1} .
\end{aligned}
$$

Herein, the stochastic parameter $p(<1)$ in step 1 linearly interpolates between $p_{0}$ and $p_{\mathrm{d}}$ if $v_{n}^{t}$ is smaller than $v_{\text {slow }}$ (note that $p$ is a real number). We set $p_{0}>p_{\mathrm{d}}$ so that step 1 is a generalization of the well known slow-tostart rule [17, 20, 21], which is known to be an ingredient in the formation of congested traffic states. Step 3 guarantees that the updating velocity satisfies the mechanical restriction as well as the traffic regulation. The stochastic deceleration in step 4 is also limited by the braking capability $D$. Note that steps 3 and 4 observe the limited deceleration consistently 22]. The length of one cell is chosen to be $\Delta x=1.5 \mathrm{~m}$ and the unit time is set to $\Delta t=1 \mathrm{sec}$. The following model parameters are motivated by empirical facts: $a=1, D=2, L=5, v_{\text {fast }}=19$, $t_{\text {safe }}=3, g_{\text {add }}=4, p_{0}=0.32, p_{\mathrm{d}}=0.11, v_{\text {slow }}=5$, and $v_{\max }=20$.

In Fig. 1 the traffic phases occurring under periodic boundary conditions are depicted. Three traffic states (free flow, synchronized traffic, jams) can be identified in the fundamental diagram Fig. 11(a) (see 2]). The straight line with the positive slope corresponds to free flow. The synchronized states form a 2-dimensional region in the middle of the diagram while jammed vehicles produce the scattered points below. A typical spatiotemporal shape of each phase is shown in Fig. I(b). Note that the socalled universal constants of traffic flow [4, 5, 6], such as the jam velocity $v_{\mathrm{g}} \approx-15 \mathrm{~km} / \mathrm{h}$ and the flux out of a jam $q_{\text {out }} \approx 1800 \mathrm{veh} / \mathrm{h}$, are also reproduced by simply 
(a)
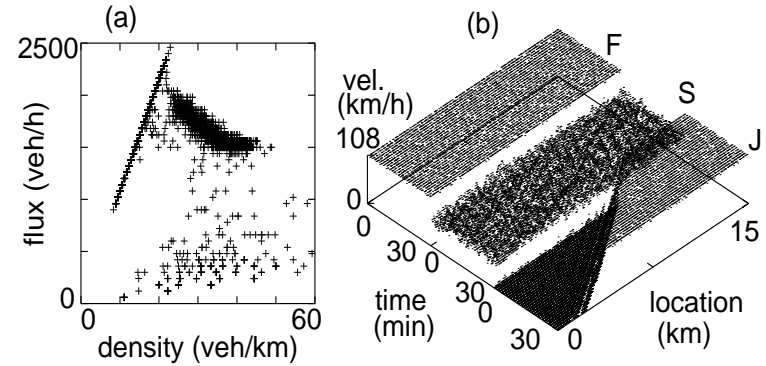

(c)

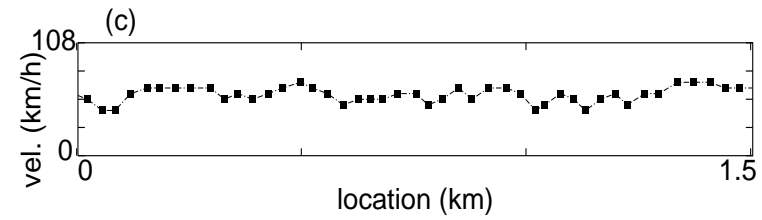

FIG. 1: Results for a road (periodic boundary conditions) consisting of 40,000 cells $(60 \mathrm{~km})$. A homogeneous distribution of standing vehicles is used as the initial condition while the density is varied from 10-50 veh $/ \mathrm{km}$ with a step of $2 \mathrm{veh} / \mathrm{km}$. (a) Fundamental diagram: Single vehicle data is gathered at a fixed position and then the flux $(J)$ and velocity $(v)$ are averaged every minute. The density $(\rho)$ is simply obtained via the hydrodynamic relation $J=\rho v$. The measuring time is 10,000 sec after the relaxation of 30,000 sec. (b) The spatiotemporal shape of the different traffic phases $[\mathrm{F}$ (ree), S(ynchronized), $\mathrm{J}$ (ammed)] is depicted for $30 \mathrm{~min}$. The initial densities are 16,30 , and $44 \mathrm{veh} / \mathrm{km}$, respectively. (c) Snapshot of synchronized flow (randomly chosen part zoomed at 40, $000 \mathrm{sec}$ ) with an initial density of $30 \mathrm{veh} / \mathrm{km}$. The filled squares represent vehicles moving from left to right.

adjusting $p_{0}$ and $p_{\mathrm{d}}$. For the purpose of demonstrating the inner structure of synchronized flow a snapshot of a part of the road is presented in Fig. 1(c). As required, this is not a transient process and exhibits a smooth velocity profile. This implies that the points comprising the synchronized area in Fig. 1(a) are not attributed to the averaged effects of strong fluctuations but to the special headway-velocity relation.

The results for an open system with on-ramp are presented in Fig. 2] We first examine the so-called pinch effect [6, 7, 18] describing the process of a local selfcompression in synchronized regions, which leads to the formation of small narrow jams. These small jams evolve finally into a few wide jams through a merging process while moving upstream when the small jams grow enough to lose the stability of the synchronized flow. This process can be seen in Fig. 2(a). Empirically, such a process is the most frequent type of congested traffic near a bottleneck and is thus named the general pattern (GP) [7]. This process is captured more clearly in Fig. 2(b). Starting from the top, it is seen that synchronized flow (high flux and slow velocity) is formed near the on-ramp. In the following two pictures, many small drops are merged into small narrow jams along the upstream. Finally, a few wide jams remain far away upstream from the on-ramp. The other known [7] types of congested traffic near bot- (a)

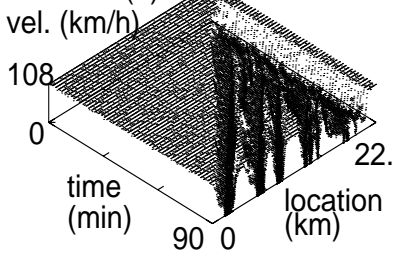

(c)

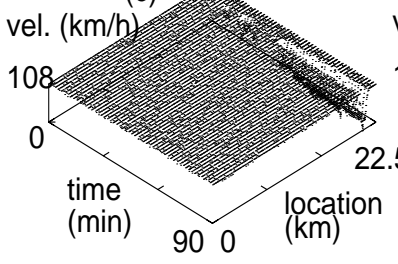

(e)
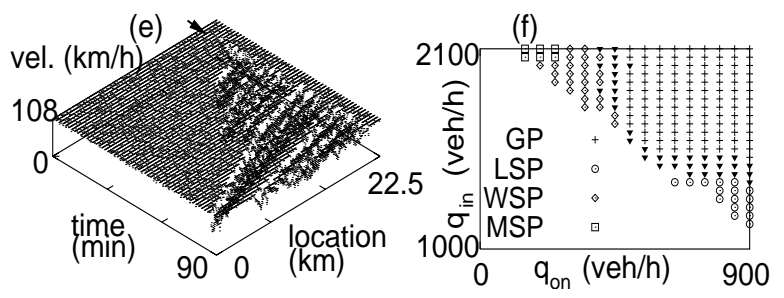

(b)

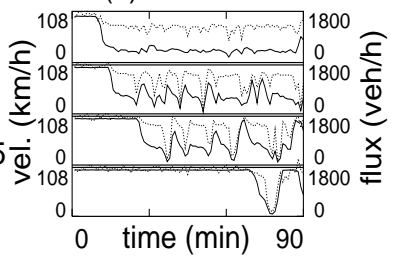

(d)
FIG. 2: The impact of an on-ramp at $20.4 \mathrm{~km}$ (black arrow) with a $0.6-\mathrm{km}$ long merging road is demonstrated. The left (right) end of the road is positioned at $-45 \mathrm{~km}(45 \mathrm{~km})$. The influx at the left is kept constant during simulation. After $q_{\text {in }}$ is supplied for 10 hours, the simulation time is set to 0 and then 8 minutes later the on-ramp injection $q_{\text {on }}$ is turned on. Within the merging road the widest leading gap is selected and then a vehicle is inserted at the mid point. For its velocity, 0.7 times that of the follower's is assigned. Below, a coupled number means $\left(q_{\text {in }}, q_{\text {on }}\right)$. (a) General pattern (GP) at $(1800,550)$. (b) Pinch effect in (a): Each figure shows one minute averaged velocity (solid line) and flux (dotted line) at different locations. From the top, detectors are located at $20.4,18.9,15.9$, and $5.4 \mathrm{~km}$, respectively. (c) Localized synchronized pattern (LSP) flow at $(1300,650)$. (d) Widening synchronized pattern (WSP) flow at $(1950,350)$. (e) Moving synchronized pattern (MSP) flow at $(2050,150)$. (f) Phase diagram of the congested traffic patterns. The filled triangles correspond to mixed patterns.

tlenecks are localized synchronized flow patterns (LSP), widening synchronized flow patterns (WSP), and moving synchronized flow patterns (MSP). It is stressed here that these patterns are also reproduced in the present model as shown in Figs 2(c,d,e). Furthermore, we remark that the phase diagram for the congested patterns [Fig. 2(f)] is quite comparable to those in [7, 18].

Finally, we examine the time-headway distribution of the model. The correspondence between the numerical results and its empirical counterpart is quite satisfactory as shown in Fig. [3 a). In contrast to the result in [17], the peaks below $1 \mathrm{sec}$ are realized merely with identical vehicles. A typical spatial configuration of the free flow shown in Fig. 31 (c) indicates that such peaks are attributed to the platoon formation of vehicles (platoon effect). Re- 
(a)

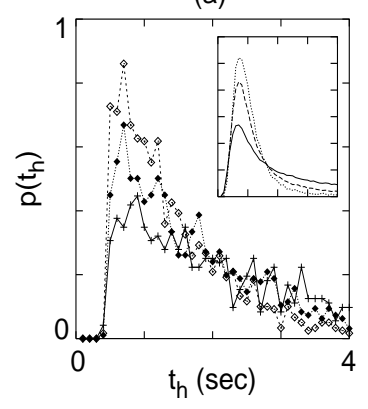

(b)

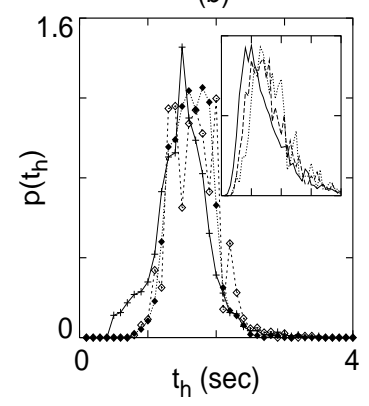

(c) platoons in free flow

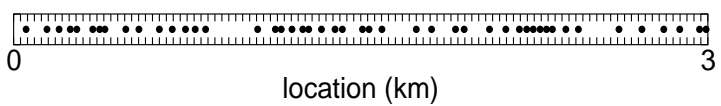

FIG. 3: (a) [(b)] Time-headway distribution of free [synchronized] flow is depicted. The cross, filled diamond, and open diamond represent densities of 12,16 , and $20 \mathrm{veh} / \mathrm{km}[24$, 32 , and $40 \mathrm{veh} / \mathrm{km}$ ]. The insets are taken from [8] (empirical data). Solid, dashed, and dotted lines correspond to density ranges from $0 \sim 12,12 \sim 24$, and $24 \sim 36$ veh $/ \mathrm{km}[24 \sim 36$, $36 \sim 48$, and $48 \sim 60 \mathrm{veh} / \mathrm{km}$ ]. (c) Randomly chosen part of the road whose overall data give the connected open diamonds in (a).

visiting Eqs. (11), (21), and (3) with the requirement of free flow $\left(c_{n}^{t+1}=v_{n+1}^{t}=v_{\max }\right.$ for all $\left.n\right)$ helps us to understand the formation of such platoons. Then Eq. (11) allows even $0.3 \mathrm{sec}$ as a time-headway, which opens up the possibility of the formation of vehicular platoons in free flow. It is emphasized here that these platoons can explain the small time-headway frequently observed in free flow without an unrealistic high flux. Thus the platoons may be one of the fundamental objects which can characterize free flow. Another important role of the platoons is suggested as follows. Since the large gaps between the platoons can absorb fluctuations propagating backwards, they stabilize free flow and thus influence the stability and transition properties. For the case of synchronized flow, qualitative agreement with empirical findings 8 is observed as follows [see Fig. (3) (b)]: i) distribution shifts to the left as density increases, ii) peaks are near $1.5 \mathrm{sec}$, iii) time-headway smaller than 1 sec still exists, and iv) large time-headway events are reduced compared to those of free flows.

In conclusion, a new CA traffic model focusing on the mechanical restriction realized by limited acceleration and braking capabilities is introduced. A further element, namely human overreaction, is implemented in order to reflect the driver's tendency toward biased reaction according to the local traffic conditions. It is shown that the model reproduces most empirical findings including the three known traffic phases, the so-called pinch effect and several types of congested traffic patterns as well as the small time-headway below 1 sec especially in free flow. In particular, the presented model produces vehic-

ular platoons as a non-trivial element of free flow. Thus the platoon effect is proposed to be the origin of many features of free flow. We remark that some situations requiring greater deceleration beyond the braking capacity for safety, such as careless insertion near on-ramp, can result in collisions in this model. In summary, it can be stressed that the presented model sheds light on open questions of traffic modelling and is therefore useful for the understanding of certain traffic phenomena as well as for applications such as fully automated driving.

H. K. L. was supported by the Brain Korea 21 Program. The authors are grateful to the BMBF within the project DAISY for financial support.

[1] B. S. Kerner, in Traffic and Granular Flow '01, edited by M. Fukui, Y. Sugiyama, M. Schreckenberg, and D. Wolf, Springer, Heidelberg, 13, (2003)

[2] D. Helbing, Rev. Mod. Phys. 73, 1067 (2001)

[3] D. Chowdhury, L. Santen, and A. Schadschneider, Phys. Rep. 329, 199 (2000)

[4] I. Treiterer and J. A. Myers, in Proceedings of the 6th International Symposium on Transportation and Traffic Theory, edited by D. J. Buckley Elsevier, New York, (1974)

[5] B. S. Kerner and H. Rehborn, Phys. Rev. E 53, R4275 (1996)

[6] B. S. Kerner, Phys. Rev. Lett 81, 3797 (1998)

[7] B. S. Kerner, Phys. Rev. E 65, 046138 (2002)

[8] W. Knospe, L. Santen, A. Schadschneider, and M. Schreckenberg, Phys. Rev. E 65, 056133 (2002)

[9] K. Nagel and M. Schreckenberg, J. Physique I 2, 2221 (1992)

[10] M. Bando, K. Hasebe, A. Nakayama, A. Shibata, and Y. Sugiyama, Phys. Rev. E 51, 1035 (1995)

[11] S. Krauss, P. Wagner, and C. Gawron, Phys. Rev. E 55, 5597 (1997)

[12] B. S. Kerner and P. Konhäuser, Phys. Rev. E 48, R2335 (1993)

[13] P. Berg, A. Mason, and A. Woods, Phys. Rev. E 61, 1056 (2000)

[14] H. K. Lee, H.-W. Lee, and D. Kim, Phys. Rev. E 64, 056126 (2001)

[15] D. Helbing, A. Hennecke, V. Shvetsov, and M. Treiber, Math. Comput. Model. 35, 517 (2002)

[16] H. K. Lee, H.-W. Lee, and D. Kim, Phys. Rev. E 69, $016118(2004)$

[17] W. Knospe, L. Santen, A. Schadschneider, and M. Schreckenberg, J. Phys. A 33, L477 (2000)

[18] B. S. Kerner and S. L. Klenov, J. Phys. A: Math. Gen. 35, L31 (2002); B. S. Kerner, S. L. Klenov, and D. E. Wolf, J. Phys. A: Math. Gen. 35, 9971 (2002)

[19] N. Eissfeldt and P. Wagner, Eur. Phys. J. B 33, 121 (2003)

[20] R. Barlovic, L. Santen, A. Schadschneider, and M. Schreckenberg, Eur. Phys. J. B. 5, 793, (1998)

[21] R. Barlovic, T. Huisinga, A. Schadschneider, and M. Schreckenberg, Phys. Rev. E 66, 046113 (2002)

[22] Limited deceleration has been examined similarly in Ref. 11] for a space continous model. However, that work 
did not take account of the possibility that its safety velocity (the counterpart of $c_{n}^{t+1}$ ) can not be realizable with limited deceleration. 